\title{
Sensitivity of dispersed-bubble flow regime identification over a broad parameter space and to various closure relations for mechanistic models of gas-liquid pipe flow
}

\author{
B. C. Houchens, F. Popa \& A. Filippov \\ Landmark, Halliburton, USA
}

\begin{abstract}
Mechanistic regime-transition functions, where one or more physical arguments are used to describe transitions, are often used to identify equilibrium multiphase flow regimes. The mechanistic models for two-phase, gas-liquid pipe flow rely on one or more closure relations, most of which have been fit to experiments of air and water systems. However, these models are often applied to problems in the oil and gas industry, which span a very broad parameter space of fluid properties. Sensitivities of the one-dimensional, two-phase, gas-liquid, mechanistic models for dispersed-bubble pipe flow are investigated over a parameter space typical of that observed in the oil and gas industry. This spans several orders of magnitude in gas density, gas and liquid viscosities, and surface tension, in addition to large ranges in superficial gas and liquid velocities, pipe diameter, and pipe inclination angle. Dispersed-bubble regime identification is most sensitive to the superficial velocities, with secondary sensitivities to densities and pipe-inclination angle in special cases.
\end{abstract}

Keywords: gas-liquid, dispersed bubble, regime identification, mechanistic.

\section{Introduction}

Multiphase regime identification is often accomplished via mechanistic regimetransition functions, where one or more physical arguments describe each possible regime transition. The transition functions are derived from a combination of: i) balances of dominant terms from the momentum equations, ii) linear-stability theory for the growth of unstable modes, and iii) simple geometric considerations related to the flow patterns. The mechanistic models 
identify the equilibrium regime and rely on one or more closure relations. Typically, these have been based on experiments of air and water systems near atmospheric pressure and room temperature. However, the models have been widely applied in the oil and gas industry to problems that span a much broader parameter space of fluid properties and operating conditions [1]. For example, in hydrocarbon production, it is possible to encounter flows where the gas has a higher viscosity than the liquid phase, or where the gas and liquid phases have similar densities.

In mechanistic modelling, consideration of the full governing mass, momentum, and energy equations are purposefully avoided because of the prohibitively high cost of their numerical solution relative to the time constraints considered acceptable for simulations in the oil and gas industry [2]. Still, several mechanistic arguments must typically be satisfied for a regime to be stable and, therefore, identified. Thus, significant effort has been committed to improving various closure relations for regime identification at all inclination angles to yield accurate, but efficient, models [3-7].

Here, the regime-identification sensitivities of the two-phase, gas-liquid, pipe-flow mechanistic models for dispersed-bubble flow are investigated over a parameter space typical of that in the oil and gas industry. Dependencies over several orders of magnitude in gas density, liquid viscosity, superficial gas and liquid velocities, surface tension, and pipe diameter, as well as pipe-inclination angle (flow direction) are spanned. The sensitivities of regime identification to the closure relations in this broad space are investigated.

\section{Problem statement}

The unified two-phase, gas-liquid, mechanistic pipe-flow model is capable of identifying eight equilibrium regimes, including dispersed bubble, bubbly (sometimes called bubble), slug, elongated bubble, churn, annular, and stratified smooth and stratified wavy flow at any inclination angle [7]. The total number of distinct flow regimes and their exact characteristics are not universally agreed upon by researchers, but these eight regimes tend to encompass all others, with the possible exceptions of mist and froth. An example of a complete regime map is given as a reference in 5 Results and discussion.

Here, only the identification of dispersed-bubble flow is considered. The regime-transition functions for dispersed-bubble flow were formulated in stages and include a buoyant creaming mechanism in horizontal flow [3], maximum packing factor and deformation-resistant limit mechanisms in vertical flow [4], and finally, a model that considered the impact of intermediate pipe-inclination angles [6]. These are summarized in the unified model given by Barnea [7].

\subsection{Model assumptions}

The mechanistic models considered are one-dimensional simplifications of inherently three-dimensional flows. Furthermore, only the equilibrium regime is identified, allowing an inherently transient problem to be treated as locally quasi- 
steady. These simplifications greatly reduce the computational modelling cost, but also require many simplifying approximations and closure relations. For example, in these one-dimensional models, counter-current flow (or counter flow) is not allowed, and the net volumetric flows of liquid and gas must be in the same direction. This allows the consideration of only positive superficial velocities, with the pipe-inclination angle specifying the flow direction.

\subsection{Nomenclature}

The space of independent parameters that determine the equilibrium two-phase, gas-liquid regime are given in Table 1. Single-phase regimes are not considered.

Table 1: Independent parameter space and limits on values considered for regime identification in two-phase, gas-liquid pipe flow.

\begin{tabular}{|c|c|c|c|c|}
\hline Symbol & Description & Minimum Value & Maximum Value & Units \\
\hline$\rho_{L}$ & liquid density & 600.0 & 1100.0 & $\left(\mathrm{~kg} / \mathrm{m}^{3}\right)$ \\
\hline$\rho_{G}$ & gas density & 0.10 & 599.0 & $\left(\mathrm{~kg} / \mathrm{m}^{3}\right)$ \\
\hline$\mu_{L}$ & liquid (dynamic) viscosity & $\begin{array}{c}2.0 \times 10^{-5} \\
(=0.02 \mathrm{cP})\end{array}$ & $\begin{array}{c}5.0 \\
(=5000.0 \mathrm{cP})\end{array}$ & $(\mathrm{Pa}-\mathrm{s})$ \\
\hline$\mu_{G}$ & gas (dynamic) viscosity & $\begin{array}{c}5.0 \times 10^{-6} \\
(=0.005 \mathrm{cP})\end{array}$ & $\begin{array}{c}5.0 \times 10^{-3} \\
(=5.0 \mathrm{cP})\end{array}$ & $(\mathrm{Pa}-\mathrm{s})$ \\
\hline$v_{S L}$ & superficial liquid velocity & $10^{-3}$ & 300.0 & $(\mathrm{~m} / \mathrm{s})$ \\
\hline$v_{S G}$ & superficial gas velocity & $10^{-3}$ & 300.0 & $(\mathrm{~m} / \mathrm{s})$ \\
\hline$\sigma_{L}$ & $\begin{array}{c}\text { surface tension of liquid in } \\
\text { contact with gas }\end{array}$ & $\begin{array}{c}0.0001 \\
(=0.1 \text { dyne } / \mathrm{cm})\end{array}$ & $\begin{array}{c}0.1 \\
(=100.0 \text { dyne } / \mathrm{cm})\end{array}$ & $(\mathrm{N} / \mathrm{m})$ \\
\hline$D$ & pipe diameter & 0.02 & 2.0 & $(\mathrm{~m})$ \\
\hline$\theta$ & $\begin{array}{c}\text { pipe-inclination angle, } \\
\text { measured from horizontal }\end{array}$ & $\begin{array}{c}/ 2 \\
\left(=-90^{\circ}\right)\end{array}$ & $\begin{array}{c}/ 2 \\
\left(=90^{\circ}\right)\end{array}$ & $(\mathrm{rad})$ \\
\hline$\varepsilon_{r}$ & pipe roughness & 0.0 & $0.05 \times D$ & $(\mathrm{~m})$ \\
\hline
\end{tabular}

The gas-void fraction, $\alpha_{G}$, is the cross-sectional area occupied by gas, $A_{G}$, divided by the entire cross-section of the pipe, $A$, given by $\alpha_{G} \equiv A_{G} / A$. The liquid holdup is the fraction of the cross-section occupied by liquid, $H_{L} \equiv A_{L} / A$, where, $\alpha_{G}+H_{L}=1$. The superficial velocities are defined in terms of the true average velocities, $v_{L}$ and $v_{G}$, as $v_{S L} \equiv v_{L} H_{L}$ and $v_{S G} \equiv v_{G} \alpha_{G}$, or in terms of the volumetric flow rates of gas and liquid, $\dot{V}_{L}$ and $\dot{V}_{G}$, as $v_{S L}=v_{L} A_{L} / A=\dot{V}_{L} / A$ and $v_{S G}=v_{G} A_{G} / A=\dot{V}_{G} / A$. The average mixture velocity, $v_{M}=\dot{V}_{G} / A+\dot{V}_{L} / A=\dot{V} / A$, can be written as $v_{M}=v_{S G}+v_{S L}$.

\section{Regime-transition functions}

The full set of gas-liquid regime-transition functions were first unified by Barnea [7] who co-developed many of them. Here, the dispersed-bubble regimetransition functions (or inequalities) for gas-liquid pipe flow are rigorously critiqued. This is not with the intention of undermining the value of this 
foundational and insightful work, but rather to remind users of their possible limitations. For a detailed review of the entire body of mechanistic multiphase modelling for all two-phase flow regimes, the reader is referred to Shoham [8]. The regime-transition functions are based on semi-mechanistic and mechanistic arguments about the flow and include geometric constraints, balances of dominant terms, linear-stability arguments, and physical arguments. The resulting regime maps are quasi-equilibrium - the duration for transition to occur is not considered.

\subsection{Distinction between dispersed and bubbly bubbles}

Dispersed-bubble flow is defined by discrete spherical gas bubbles in a liquid matrix, where the bubbles do not interact. It is assumed that the trajectories of dispersed bubbles are determined only by the liquid matrix; in equilibrium, they are simply convected with the liquid and do not slip relative to it. Note that this can be roughly true in an equilibrium sense even when the distribution of bubbles is not uniform, such as in horizontal flow where larger dispersed bubbles are more likely found toward the top of the pipe, but where the buoyant force is still sufficiently small that it does not cause the bubbles to interact.

\subsection{Transition to and from dispersed-bubble flow to all other regimes}

If the dispersed bubbles begin to interact, either by modifying the trajectories of neighbouring bubbles, by coalescence, or if they deform, the regime is no longer dispersed-bubble. The regime-transition functions that identify dispersed-bubble flow are presented in the subsequent sections.

\subsubsection{Maximum-allowable dispersed-bubble diameter}

The mechanism of bubble breakup is taken to be a balance between turbulent fluctuations and surface-tension forces $[9,10]$. This leads to the prediction of a maximum dispersed-bubble (DB) diameter, $d_{D B \text {, max }}$, that is stable (break-up resistant) for a specified level of turbulence given by:

$$
d_{D B \text {, max }}=\left[k_{\text {vert }}+4.15\left(\frac{v_{S G}}{v_{M}}\right)^{1 / 2}\right]\left(\frac{\sigma_{L}}{\rho_{L}}\right)^{3 / 5}\left(\frac{2 f_{D B} v_{M}^{3}}{D}\right)^{-2 / 5},
$$

where $k_{\text {vert }}$ was determined to be 0.725 by Hinze [9] who fit to the data of Clay [11] for various fluid combinations, including water and kerosene, and $f_{D B}$ is the Fanning friction factor based on the superficial mixture velocity and various closure relations for the viscosity and density. It should be noted that the data used to fit $k_{\text {vert }}$ is from flow between coaxial cylinders, with the inner cylinder rotating. The standard deviation in the fit of $k_{\text {vert }}=0.725$ was 0.315 , though the trend is quite good over several decades of data in both drop size and energy input. The impact of the choice of $f_{D B}$ is discussed later. In addition, the factor of $4.15\left(v_{S G} / v_{M}\right)^{1 / 2}$ was not included in the original 1980 work of Taitel et al. [4] 
but was added by Barnea et al. [5], later claiming it to be a correction for flows other than those that are vertically upward [6]. In reality, this is a match to Calderbank's data [12] on agitation experiments, not pipe-flow experiments, which only considered $\alpha_{G}$ up to approximately $8 \%$, much lower than the maximum possible value for dispersed-bubble flow. Thus, it is unclear how justified Barnea was in matching this data, which she pointed out herself [5].

\subsubsection{Maximum packing factor for stable dispersed-bubble flow}

Taitel et al. [4] assumed that a gas-void fraction of $\alpha_{G}=0.52$ gives the maximum packing factor of dispersed gas bubbles in liquid. This is the simple-cubic packing factor for spheres. It is assumed that for $\alpha_{G} \geq 0.52$, coalescence occurs even in strong turbulence due to the close proximity of the bubbles. When the bubbles are packed tightly, they tend to deform each other, leading to more chaotic trajectories and then coalescence. At the point of interaction, the bubbles are no longer isolated and the regime cannot be considered dispersed bubble. Because dispersed bubbles are small, they are assumed to not slip relative to the liquid matrix, and $\alpha_{G} \rightarrow \alpha_{G_{N S}}$, where NS indicates no-slip. From the steady-state conservation of mass, it can be shown that:

$$
\alpha_{G_{N S}}=\frac{v_{S G}}{v_{S L}+v_{S G}}=\frac{v_{S G}}{v_{M}} .
$$

Thus, a necessary, but not sufficient, condition for stable dispersed-bubble flow is:

$$
\alpha_{G_{N S}}<0.52
$$

for any inclination angle.

\subsubsection{Maximum diameter for deformation-resistant dispersed bubbles}

If the turbulent breakup produces sufficiently small bubbles that remain spherical and non-interacting, and the turbulence additionally prevents coalescence, then the dispersed-bubble flow will be stable. Dispersed bubbles will remain undeformed and, thus, coalescence-resistant when they are smaller than:

$$
d_{C D}=2\left[\frac{0.4 \sigma_{L}}{\left(\rho_{L}-\rho_{G}\right) g}\right]^{1 / 2},
$$

where the subscript CD refers to the "critical-deformation" diameter limit. This is modified from Brodkey's reported value [13, p. 584], which was originally derived by Bond and Newton [14] from dimensional arguments and did not include the factor of 2. While Bond and Newton associated this limit with circulation, Brodkey points out that the departure from "solid-like" behaviour of bubbles is actually a result of bubble distortion and swerving motion. This is an Eötvös number scaling argument, with the Eötvös number set to roughly order one. The surface tension acts to stabilize the spherical shape of the dispersed bubbles, while the density difference promotes deformation. Barnea et al. [15] added the factor of 2 to better match to the data of Miyagi [16], though that 
factor was not used in the original mechanistic modelling by Taitel et al. [4]. This mechanistic argument assumes that $\rho_{L}>\rho_{G}$. Thus, when:

$$
d_{D B, \max }<d_{C D}
$$

dispersed-bubble flow is allowed based on this regime transition, which is the second necessary, but not sufficient, condition for stable dispersed-bubble flow.

\subsubsection{Maximum diameter before buoyant creaming of dispersed bubbles}

The final regime-transition inequality for dispersed-bubble flow occurs as a result of a balance between buoyant forces and turbulent fluctuations [3] and finds relevance for horizontal, near-horizontal, and intermediate-inclination angles. If the buoyant forces dominate, then the gas bubbles will migrate to the top of the pipe faster than they can be re-dispersed into the liquid phase. The bubbles will interact and coalesce near the top of the pipe, leading to creaming and transition to either intermittent or stratified flow. The balance between buoyant and turbulent forces yields the critical bubble diameter, $d_{C B}$, above which bubbles will migrate to the top of the pipe faster than they are re-dispersed by turbulence, where subscript CB refers to "critical buoyant."

The component of the net buoyant force on a bubble of diameter, $d_{B}$, in the direction normal to the pipe axis is given by:

$$
F_{B, \text { net }}=\left(\rho_{L}-\rho_{G}\right) g \cos \theta \frac{\pi d_{B}^{3}}{6},
$$

which serves to draw the dispersed bubbles toward the top of the pipe when the inclination angle is anything other than $\pm 90^{\circ}$. The opposing force due to turbulent fluctuations was given by Levich [17] as:

$$
F_{T}=\frac{1}{2} \rho_{L} \underbrace{\frac{v_{M}^{2} f_{D B}}{2}}_{\substack{\text { square of radial } \\ \text { vel. fluctuations }}} \frac{\pi d_{B}^{2}}{4},
$$

where Taitel and Dukler [3] proposed that the root-mean square of the radialvelocity fluctuations could be approximated by the friction velocity, which can be represented by $v_{M}\left(f_{D B} / 2\right)^{1 / 2}$. The Fanning friction factor, $f_{D B}$, is based on the mixture velocity, as discussed for eqn (1), and the choice of friction factor and mixture property models. Then, when $F_{B, \text { net }}>F_{T}$, bubbles will migrate to the top of the pipe, and the flow will transition from dispersed bubble to intermittent. The critical bubble diameter occurs when these forces are equated, giving:

Thus, when

$$
d_{C B}=\frac{3}{8}\left[\frac{\rho_{L}}{\rho_{L}-\rho_{G}}\right] \frac{f_{D B} v_{M}^{2}}{g \cos \theta} .
$$

$$
d_{D B, \max }<d_{C B}
$$

dispersed-bubble flow is allowed based on this third regime-transition function, a necessary, but not sufficient, condition for stable dispersed-bubble flow. To allow evaluation at all pipe-inclination angles, eqn (9) is multiplied by $\cos \theta$, giving: 


$$
d_{D B, \max } \cos \theta<d_{C B} \cos \theta .
$$

Regime-transition functions (3), (5) and (10) determine the boundaries for stable dispersed-bubble flow at all pipe inclinations. Each is a necessary, but not sufficient, condition for the existence of dispersed-bubble flow. Collectively, they are necessary and sufficient conditions.

\section{Closure relations}

To evaluate $f_{D B}$ in eqn (1) for $d_{D B \text {, max }}$ and eqn (8) for $d_{C B}$ requires the evaluation of a dispersed-bubble Reynolds number, $R e_{D B}=\rho_{D B} v_{D B} D / \mu_{D B}$, which, in turn, requires models for the mixture density, $\rho_{D B}$, and mixture viscosity, $\mu_{D B}$. The mixture density is appropriately evaluated as a volume-weighted average, given by $\rho_{D B}=\rho_{M, v o l}=\alpha_{G} \rho_{G}+H_{L} \rho_{L}$, arising from $\rho_{M, v o l}=m / \forall=\rho_{G} V_{G} / \forall+\rho_{L} V_{L} / V$, where $m$ is the total mass of the mixture in a volume, $\forall$, and where $V_{G} / \forall$ and $V_{L} / \forall$ evaluate to the same values as $A_{G} / A$ and $A_{L} / A$, respectively, due to the one-dimensional nature of the flow. While commonly used, this is not universal. For example, Taitel et al. [4] instead used $\rho_{D B}=\rho_{L}$, as discussed further below.

\subsection{Mixture viscosity}

A commonly used model for mixture viscosity is a volume-weighted average, given by $\mu_{D B}=\mu_{M, v o l}=\alpha_{G} \mu_{G}+H_{L} \mu_{L}$ (see eqn 2.11 in Shoham [8]). However, if the viscosities have very different magnitudes, the physical justification for this model is weak. In dispersed-bubble flow, where liquid is expected to be in contact with the pipe walls, the massive reduction in viscosity due to passive gas bubbles seems overstated, particularly as $\alpha_{G}$ approaches 0.52 . Alternatively, the mixture viscosity can be set to that of the matrix (liquid) viscosity (as used by Taitel et al. [4]), or to a mass-weighted average, $\mu_{M, \text { mass }}=x_{G} \mu_{G}+\left(1-x_{G}\right) \mu_{L}$, where $x_{G}$ is the gas quality. The impact of each of these models will be shown.

\subsection{Friction factor}

The exact Fanning friction factor, $f_{D B, \text { lam }}=16 R e_{D B}^{-1}$, is always used for $R e_{D B}<2300$ based on the analytical solution for single-phase flow. In mechanistic modelling, the turbulent Fanning friction factor is often evaluated using the Blasius form, $f_{D B, \text { Blas }}=C_{D B}\left(\rho_{D B} v_{M} D / \mu_{D B}\right)^{-n_{D B}}$ (see for example [3-8]). This has the same form as $f_{D B \text {,lam }}$ but does not account for variable pipe roughness. Taitel and Dukler [3] and Taitel et al. [4] used the Blasius form, took the mixture properties as that of the liquid $\left(\mu_{D B}=\mu_{L}\right.$ and $\left.\rho_{D B}=\rho_{L}\right)$, and set $C_{D B, \text { turb }}=0.046$ and $n_{D B, \text { turb }}=0.2$. For dispersed-bubble flow, it is expected that primarily liquid would be in contact with the pipe wall, making the choice of $\mu_{D B}=\mu_{L}$ appear reasonable. However, the mixture density would be expected to be significantly modified by the presence of gas. 
Alternatively, the Haaland [18] approximation to the Colebrook equation is an explicit relationship for the turbulent Fanning friction factor given by:

$$
f_{D B, \text { Haal , turb }}=\frac{1}{4}\left[-1.8 \log _{10}\left[\left(\frac{\varepsilon_{r} / D}{3.7}\right)^{1.11}+\frac{6.9}{R e_{D B}}\right]\right]^{-2} \text {. }
$$

Both friction-factor models are smoothed between laminar and turbulent flow.

\section{Results and discussion}

Here it is assumed that the mechanistic models are perfectly valid, and only the impacts of the closure relations are considered. This is a significant assumption, as there are several questionable, but accepted, empirically based values included in the regime-transition functions. The most notable include the factor in brackets in eqn (1) used to evaluate $d_{D B \text {, max }}$, the assumed maximum packing density of 0.52 appearing in eqn (3), the inserted factor of 2 in eqn (4), and the approximation of the root-mean square of the radial-velocity fluctuations by the friction velocity, represented by $v_{M}\left(f_{D B} / 2\right)^{1 / 2}$ in eqn (8). All of these are taken as correct, and only the influences of a broad parameter space, mixture-property closure relations, and friction-factor models are considered.

As a baseline, the regime map for air and water at $20^{\circ} \mathrm{C}$ and 1 atmosphere is presented in Figure 1 for properties $\rho_{L}=998 \mathrm{~kg} / \mathrm{m}^{3}, \quad \rho_{G}=1.204 \mathrm{~kg} / \mathrm{m}^{3}$, $\mu_{L}=1.002 \times 10^{-3} \mathrm{~kg} / \mathrm{m}-\mathrm{s}, \mu_{G}=1.825 \times 10^{-5} \mathrm{~kg} / \mathrm{m}-\mathrm{s}$, and $\sigma_{L}=0.073 \mathrm{~N} / \mathrm{m}$ and with superficial velocities varied over the ranges $0.001 \leq v_{S L} \leq 300 \mathrm{~m} / \mathrm{s}$ and $0.001 \leq v_{S G} \leq 300 \mathrm{~m} / \mathrm{s}$ for a smooth pipe $\left(\varepsilon_{r}=0\right)$ with diameter $D=0.1 \mathrm{~m}$ and vertical-upward inclination angle $\theta=90^{\circ}$.

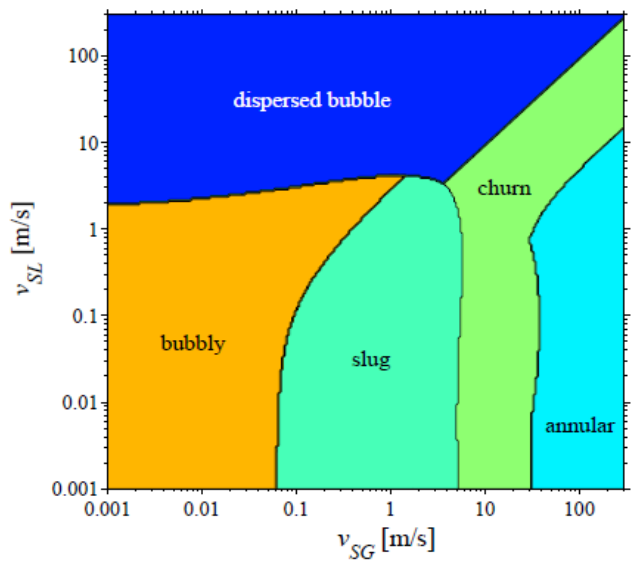

Figure 1: $\quad$ Regime map for air and water at $20^{\circ} \mathrm{C}$ and 1 atm for a smooth pipe with $D=0.1 \mathrm{~m}, \theta=90^{\circ}, \rho_{D B}=\rho_{M, v o l}, \mu_{D B}=\mu_{M, \text { vol }}$, and $f_{D B, \text { Haal turb }}$. 


\subsection{Influence of superficial velocities on dispersed-bubble identification}

The regime-transition functions that identify dispersed-bubble flow, given by eqns (3), (5) and (10), all depend on $v_{S L}$ and $v_{S G}$. Eqns (5) and (10) both depend directly on $\rho_{L}, \rho_{G}, \sigma_{L}$, and $D$. Only eqn (8) depends on $\theta$. The roles played by $\mu_{L}, \mu_{G}$, and $\varepsilon_{r}$ in dispersed-bubble-flow identification are only through $f_{D B}$.

The impact of $v_{S L}$ and $v_{S G}$ is relatively straightforward and independent of the fluid combination. Together, eqns (2) and (3) require $0.52\left(v_{S L}+v_{S G}\right)>v_{S G}$ for dispersed-bubble flow to exist. Stated another way, dispersed-bubble flow might exist (necessary, but not sufficient) if $v_{S L} \gtrsim v_{S G}$, which is consistent with a liquiddominated flow containing discrete gas bubbles. The superficial velocities also directly impact $d_{D B \text {, max }}$, and, thus, the regime-transition functions given by eqns (5) and (10). As the superficial velocities increase, the associated turbulence is better able to break up the bubbles, leading to smaller values of $d_{D B \text {,max }}$. This is shown in Figure 2 for air and water at $20^{\circ} \mathrm{C}$ and $1 \mathrm{~atm}$, with superficial velocities varied over the reduced ranges $0.1 \leq v_{S L} \leq 300 \mathrm{~m} / \mathrm{s}$ and $0.1 \leq v_{S G} \leq 300 \mathrm{~m} / \mathrm{s}$ for a smooth pipe $\left(\varepsilon_{r}=0\right)$ with diameter $D=0.1 \mathrm{~m}$. Note that $d_{D B \text {, } \max }$ is independent of inclination angle. Also, at and below $v_{S L}=v_{S G}=0.1 \mathrm{~m} / \mathrm{s}$, the allowable $d_{D B \text {, } \max }$ is larger than the pipe diameter, $D$, so the lower limits of $v_{S L}$ and $v_{S G}$ are truncated in Figure 2. In this case, the domain of allowed dispersed-bubble flow would be limited by the regime-transition functions of eqns (5) and (10).

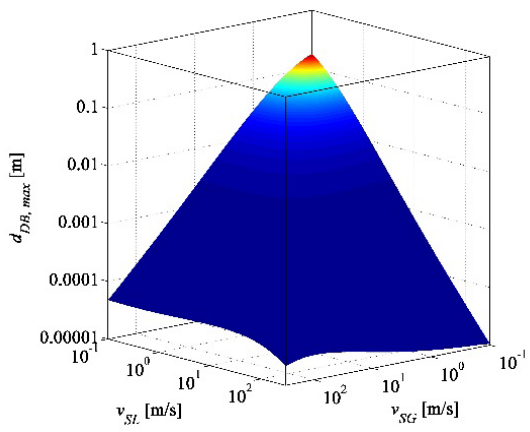

Figure 2: Variation of $d_{D B, \max }$ with $v_{S L}$ and $v_{S G}$ for air and water at $20^{\circ} \mathrm{C}$ and 1 atm, for a smooth pipe with $D=0.1 \mathrm{~m}$ and closure models $\rho_{D B}=\rho_{M, v o l}, \mu_{D B}=\mu_{M, v o l}$, and $f_{D B, \text { Haal }, \text { turb }}$ used (note the directions of the axes).

\subsection{Influence of densities on dispersed-bubble identification}

Gas and liquid densities can be very similar in oil and gas applications. As $\rho_{G}$ and $\rho_{L}$ approach, both $d_{C D}$ and $d_{C B}$ increase, as shown by eqns (4) and (8). 
As $\rho_{G} \rightarrow \rho_{L}$, the regime-transition functions given by eqns (5) and (10) will be satisfied, and dispersed-bubble flow will fill all of the domain that satisfies $v_{S L} \geq v_{S G}$. The dispersed-bubble regime map is shown in Figure 3 for three density combinations. Note the left-most figure uses the same inputs from the regime map in Figure 1. Though the trend for the domain to be increasingly filled with dispersed-bubble flow as $\rho_{G} \rightarrow \rho_{L}$ does hold, it is in practice only realized when $\rho_{G}$ very closely approaches $\rho_{L}$.
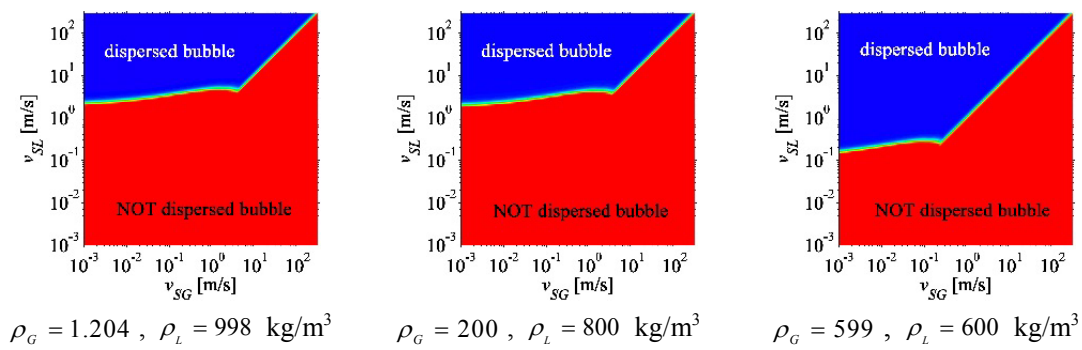

Figure 3: Dispersed-bubble regime map in the superficial velocity space, with various density combinations, and with $\mu_{L}, \mu_{G}$, and $\sigma_{L}$ fixed as the properties of air and water at $20^{\circ} \mathrm{C}$ and $1 \mathrm{~atm}$, with the closure models $\rho_{D B}=\rho_{M, \text { vol }}, \mu_{D B}=\mu_{M, \text { vol }}$, and $f_{D B, \text { Haal, turb }}$ used.

\subsection{Influence of surface tension and inclination angle on dispersed-bubble identification}

Both $d_{D B, \max }$ and $d_{C D}$ depend on surface tension, but together in the regimetransition function given by eqn (5) they almost negate each other, having a net effect given by $\sigma_{L}^{3 / 5} \sigma_{L}^{-1 / 2}=\sigma_{L}^{1 / 10}$. Over the three orders of magnitude range of allowable $\sigma_{L}$, the value of $\sigma_{L}^{1 / 10}$ varies only from 0.4 to 0.8 .

Furthermore, because $d_{C D}$ is independent of $\theta$, the only significant dependence on $\sigma_{L}$ and $\theta$ occurs when the buoyant creaming mechanism $\left(d_{C B}\right)$ controls the boundary of the dispersed-bubble regime expected to occur for nearhorizontal flow. This transition mechanism is rarely controlling. However, such a case is shown by selecting properties that might be observed in an oil and gas well of $\rho_{L}=650 \mathrm{~kg} / \mathrm{m}^{3}, \mu_{L}=2.0 \times 10^{-4} \mathrm{~kg} / \mathrm{m}-\mathrm{s}, \rho_{G}=1.0 \mathrm{~kg} / \mathrm{m}^{3}, \mu_{G}=2.0 \times 10^{-5}$ $\mathrm{kg} / \mathrm{m}-\mathrm{s}$, and varying the inclination angle and surface tension for three combinations of superficial velocities. The combined regime-transition functions for dispersed-bubble flow are plotted in Figure 4 in the surface-tension inclination-angle space for a smooth pipe $\left(\varepsilon_{r}=0\right)$ with $D=0.1 \mathrm{~m}$. From left to right, the transition is controlled by just buoyant creaming $\left(d_{C B}\right)$, then a mixture of buoyant creaming $\left(d_{C B}\right)$ and bubble distortion $\left(d_{C D}\right)$, and finally by $d_{C D}$ alone. 

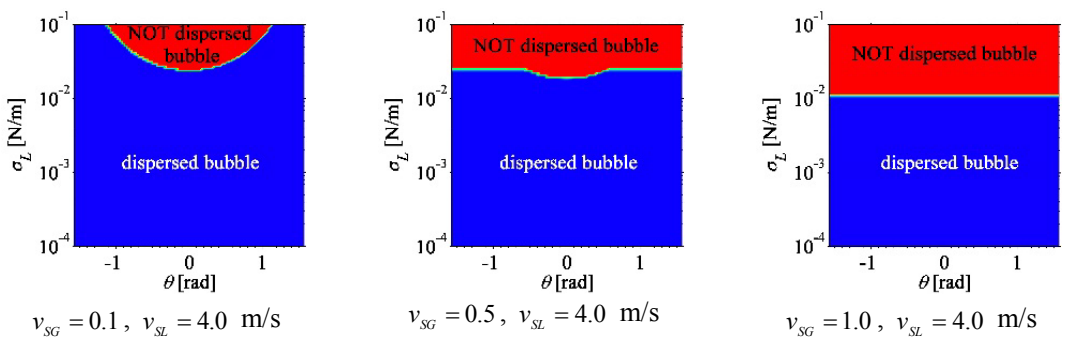

Figure 4: Dispersed-bubble regime map in the $\theta-\sigma_{L}$ space, with $\rho_{L} \mu_{L}, \rho_{G}$, and $\mu_{G}$ fixed at possible oil and gas properties, and with the closure models $\rho_{D B}=\rho_{M, v o l}, \mu_{D B}=\mu_{M, v o l}$, and $f_{D B, \text { Haal, turb }}$ used.

\subsection{Combined influence of the mixture density, mixture viscosity, and friction-factor models on dispersed-bubble identification}

For large $v_{S L}$, the dispersed-bubble regime exists over a wide range of $v_{S G}$. Four mixture density and mixture viscosity combinations are considered: the original form of Taitel et al. $\left(\rho_{D B}=\rho_{L}, \mu_{D B}=\mu_{L}\right)[3,4]$, and three others, which take $\rho_{D B}=\rho_{M, v o l}$ with either $\mu_{D B}=\mu_{L}$ or $\mu_{D B}=\mu_{M, v o l}$ or $\mu_{D B}=\mu_{M, \text { mass }}$. Sensitivities are demonstrated well by air and water at $20^{\circ} \mathrm{C}$ and $1 \mathrm{~atm}$, since their base densities and viscosities are orders of magnitude apart. Consider vertically upward flow with $v_{S L}=10 \mathrm{~m} / \mathrm{s}$, which places all $R e_{D B}$ well within the turbulent range. The value of $R e_{D B}$ directly impacts $f_{D B}$, which impacts $d_{D B \text {, max }}, d_{C D}$ and $d_{C B}$. However, a high $v_{S L}$ will satisfy the regime transitions given by eqns (5) and (10), and only when $\alpha_{G_{N S}}<0.52$ is violated does dispersed-bubble flow cease to exist. Thus, with respect to $v_{S G}$ or $\alpha_{G}$, all parameters can be plotted over the same limits. The impacts on $R e_{D B}$ and $f_{D B \text {, Blas }}$ due to the density and viscosity models are shown versus $v_{S G}$ in Figure 5 . When the density and viscosity models are fixed, the maximum error between the Haaland and Blasius forms for air and water at $20^{\circ} \mathrm{C}$ and $1 \mathrm{~atm}$ is $2.1 \%$, and thus, only $f_{D B \text {, Blas }}$ is plotted.

The cases $\left(\rho_{D B}=\rho_{L}, \mu_{D B}=\mu_{L}\right)$ and $\left(\rho_{D B}=\rho_{M, v o l}, \mu_{D B}=\mu_{M, v o l}\right)$ produce very similar $\operatorname{Re}_{D B}$ and, therefore, similar friction factors. For the latter, the net effects of volume averaging both the density and viscosity in $\operatorname{Re}_{D B}=\rho_{D B} v_{D B} D / \mu_{D B}$ offset. However, since $\rho_{D B}=\rho_{M, v o l}$ is a much better approximation to the mixture density, this is the preferred method of the two, particularly if the hydrostatic component of pressure drop is of interest.

Likewise, the cases $\left(\rho_{D B}=\rho_{M, v o l}, \mu_{D B}=\mu_{L}\right)$ and $\left(\rho_{D B}=\rho_{M, v o l}, \mu_{D B}=\mu_{M, \text { mass }}\right)$ produce similar $R e_{D B}$ and $f_{D B}$, since the density model is the same and the viscosity model of the latter is dominated by the liquid contribution, which 

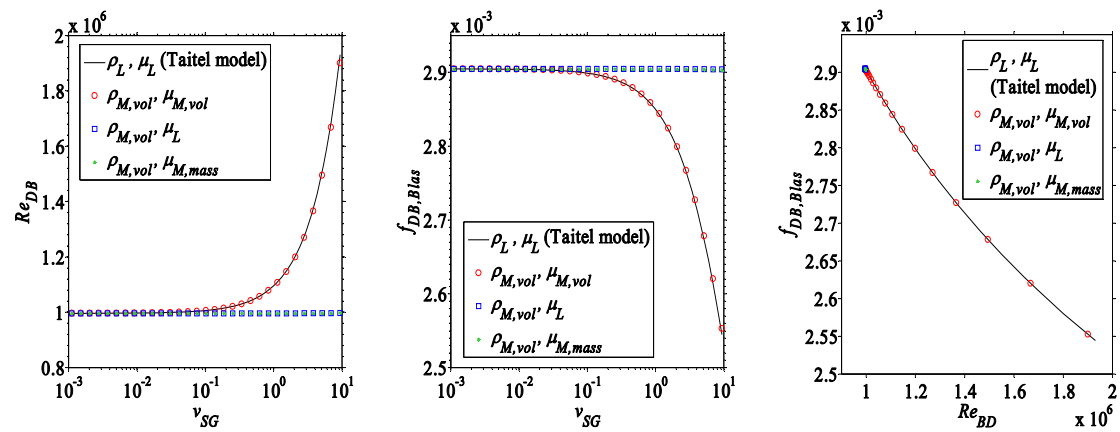

Figure 5: $\quad R e_{D B}$ and $f_{D B, B l a s}$ dependence on closure models for mixture density and viscosity, over ranges where dispersed-bubble flow exists.

accounts for most of the mass. This trend will not hold if the densities are similar but the viscosities very different. These latter two models predict $f_{D B}$ up to $12.4 \%$ higher than the cases above with $\left(\rho_{D B}=\rho_{L}, \mu_{D B}=\mu_{L}\right)$ and $\left(\rho_{D B}=\rho_{M, v o l}\right.$, $\mu_{D B}=\mu_{M, v o l}$ ), with the greatest differences occurring near the upper limit of $v_{S G}$. However, the regime transition for large $v_{S G}$ is controlled primarily by $\alpha_{G_{N S}}<0.52$, which is not impacted by $f_{D B}$. Thus, the closure models for mixture density and mixture viscosity have very little influence on dispersed-bubble regime identification. Note, however, that the frictional component of pressure drop is directly proportional to $f_{D B}$.

\section{Conclusions}

For oil and gas flows, the regime-transition functions for dispersed-bubble flow can vary significantly from that for air and water. The dominant sensitivity of regime identification is to the superficial velocities. Secondary sensitivities include the density difference, especially when $\rho_{G} \rightarrow \rho_{L}$, and the inclination angle for cases where $d_{D B \text {, max }}<d_{C B}$ controls the regime transition.

For pressure gradient, the roles played by the combined density and viscosity models significantly impact the friction factor and associated frictional component of pressure gradient. The mixture density for dispersed-bubble flow is directly proportional to both the frictional and gravitational components.

\section{References}

[1] Gomez, L.E., Shoham, O., Zelimir, S., Chokshi, R.N. \& Northug, T., Unified mechanistic model for steady-state two-phase flow: Horizontal to vertical upward flow. SPE Journal, 5(3), pp. 339-350, 2000. 
[2] Taitel, Y. Advances in two phase flow mechanistic modeling. SPE paper 27959 presented at the University of Tulsa Centennial Petroleum Engineering Symposium, Tulsa, Oklahoma, 29-31 August 1994.

[3] Taitel, Y. \& Dukler, A.E., A model for predicting flow regime transitions in horizontal and near horizontal gas-liquid flow. AIChE Journal, 22(1), pp. 47-55, 1976.

[4] Taitel, Y., Barnea, D. \& Dukler, A.E., Modelling flow pattern transitions for steady upward gas-liquid flow in vertical tubes. AIChE Journal, 26(3), pp. 345-354, 1980.

[5] Barnea, D., Shoham, O. \& Taitel, Y., Flow pattern transition for downward inclined two phase flow: Horizontal to vertical. Chemical Eng. Sci., 37(5), pp. 735-740, 1982a.

[6] Barnea, D., Transition from annular flow and from dispersed bubble flow unified models for the whole range of pipe inclinations. Int. J. Multiphase Flow, 12(5), pp. 733-744, 1986.

[7] Barnea, D., A unified model for predicting flow-pattern transitions for the whole range of pipe inclinations. Int. J. Multiphase Flow, 13(1), pp. 1-12, 1987.

[8] Shoham, O., Mechanistic Modeling of Gas-liquid Two-Phase Flow in Pipes, Society of Petroleum Engineers, 2006.

[9] Hinze, J.O., Fundamentals of the hydrodynamic mechanism of splitting in dispersion processes. AIChE Journal, 1(3), pp. 289-295, 1955.

[10] Sevik, M. \& Park, S.H., The splitting of drops and bubbles by turbulent fluid flow. J. Fluids Engineering, pp. 53-60, 1973.

[11] Clay, P.H., The mechanism of emulsion formation in turbulent flow. I. Experimental part. Proc. R. Acad. Sci. (Amsterdam), 43, pp. 852-865, 1940.

[12] Calderbank, P.H., Physical rate processes in industrial fermentation, Part I: The interfacial area in gas-liquid contacting with mechanical agitation. Trans. Instn. Chem. Engrs. 36, pp. 443-463, 1958.

[13] Brodkey, R.S., The Phenomena of Fluid Motion, Addison-Wesley Publishing, Boston, 1967. (Reprinted by Dover, 1995.)

[14] Bond, W.N. \& Newton, D.A., Bubbles, drops, and Stoke's law. Phil. Mag., pp. 794-800, 1928.

[15] Barnea, D., Shoham, O. \& Taitel, Y., Flow pattern transition for vertical downward two phase flow. Chemical Eng. Sci., 37(5), pp. 741-744, $1982 b$.

[16] Miyagi, O., The motion of an air bubble rising in water. Phil. Mag., pp. 112-140, 1925.

[17] Levich, V.G., Physicochemical Hydrodynamics, Prentice-Hall, Englewood Cliffs, NJ, 1962.

[18] Haaland, S.E., Simple and explicit formulas for the friction factor in turbulent pipe flow. J. Fluids Engineering, pp. 89-90, 1983. 